\title{
ELEMENTOS NORTEADORES PARA A APRESENTAÇÃO DE CONCEITOS ELEMENTARES DE GEOMETRIA ESFÉRICA E HIPERBÓLICA À ESTUDANTES DO ENSINO MÉDIO
}

\author{
Wanderley Pivatto Brum ${ }^{1}$ \\ Elcio Schuhmacher ${ }^{2}$
}

Resumo: O presente artigo constitui-se uma discussão teórica a respeito da apresentação de conceitos elementares de Geometria Esférica e Hiperbólica no Ensino Médio. Tal conteúdo de estudo, ainda não obrigatório no ensino escolar, constitui-se elemento importante do saber específico do futuro professor de Matemática e dos estudantes. Tem como objetivo, analisar o posicionamento dos Parâmetros Curriculares Nacionais para o desenvolvimento do pensamento geométrico não euclidiano, apontar alguns entraves para sua inserção no Ensino Médio e apresenta, por fim, justificativas para sua apresentação na sala de aula. O estudo, por fim, relembra que a Geometria Euclidiana tem sido apresentada na escola básica como a única existente, quando de fato é apenas uma pequena parte das Geometrias, pois existem outras, mais amplas, que, muitas vezes, são completamente desconhecida dos estudantes e, até mesmo ignorada pelos professores.

Palavras-chave: Geometria esférica. Geometria hiperbólica. Conceitos elementares. Ensino de Matemática.

\begin{abstract}
This paper constitutes a theoretical discussion about the presentation of basic concepts of spherical geometry and hyperbolic in high school. This study content not yet mandatory in high school, it constitutes an important element of the specific knowledge of the future mathematics teacher and students. Aims to analyze the positioning of the National Curriculum for the development of non-Euclidean geometric thinking, point out some obstacles to their integration in high school and has finally justifications for its presentation in the classroom. The study finally recalls that Euclidean geometry has been presented in the elementary school as the only one, when in fact it is only a small part of the Geometries, because there are other, broader, which often are completely unknown to the students and even ignored by teachers.
\end{abstract}

Keywords: spherical Geometry. hyperbolic Geometry. elementary Concepts. Mathematics teaching.

\footnotetext{
1 Mestre em Ensino de Ciências Naturais e Matemática. Programa de Pós - graduação em Ensino de Ciências Naturais e Matemática - FURB Rua Antônio da Veiga, 140, Victor Konder, 89012-900 Blumenau (SC), 32637441, ufsc2013@yahoo.com.br.

2 Dr. em Química pela Universidade Federal de Santa Catarina. Programa de Pós - graduação em Ensino de Ciências Naturais e Matemática - FURB Rua Antônio da Veiga, 140, Victor Konder, 89012-900 Blumenau (SC), 32210637, elcio@,furb.br.
} 


\section{Introdução}

Tradicionalmente, os conhecimentos geométricos abordados em sala de aula se restringem às relações lógicas e construções de símbolos advindos de uma Geometria dedutiva e axiomática, estabelecida na Grécia há cerca de 2700 anos e conhecida hoje como Geometria Euclidiana. No entanto, ao observar a superfície terrestre com sua forma elipsoidal, as montanhas, as ondas do mar e tantos outros objetos encontrados na natureza, evidenciam-se as dificuldades de construir alguns conceitos no campo da Geometria sustentados por noções primitivas como de reta, ponto e plano.

Nas duas últimas décadas, houve uma intensa discussão nos meios educacionais por parte dos membros de associações de profissionais da Matemática, como Kalleff (2004), Cabariti (2006), Alves (2008), Bongiovani (2010), Carvalho e Carvalho (2011), Cedrez (2012), Leivas (2012) e Martos (2012) para a inclusão de conteúdos advindos da Geometria não Euclidiana, como a Geometria Esférica e Hiperbólica nos bancos escolares, considerada como adequado à formação de estudantes para o século XXI em decorrência dos avanços teóricos da Matemática e da Computação. Dessas discussões emergiram alguns questionamentos: o ensino da Geometria não Euclidiana é um tema distante da realidade dos estudantes? O modelo geométrico para representar o planeta Terra abordado nas aulas de Geografia e Matemática é um plano, uma folha retangular ou uma superfície quase esférica? Se um dos pontos de discussão é a reformulação do ensino no Brasil, porque as Geometrias não Euclidianas ainda não são consideradas um ramo importante da Matemática por parte dos professores? Será que o ensino de Geometrias não Euclidianas, como a Esférica e Hiperbólica vem causando inquietações em professores de Matemática por ser um assunto novo e ainda desconhecido?

A busca de respostas para estas questões revela além de sua importância, a necessidade de divulgação junto aos professores desse conteúdo ainda pouco explorado em sala de aula, afinal, nos dias atuais, naves espaciais, por exemplo, percorrem em suas viagens trajetórias que não são retilíneas e no passado, segundo Eves (2008), Boyer (2009) e Mlodinow (2010) existem registros que indicam o medo de alguns navegadores por acreditarem que ao fim do horizonte, navios eram engolidos ou chegaria ao "fim do mundo". Este pensamento era devido à interpretação que muitas pessoas atribuíam ao formato da Terra. 


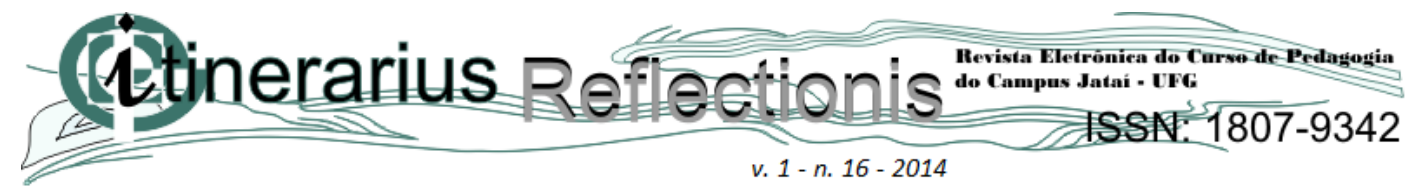

É possível encontrar, ainda, alguns estudantes que representam geometricamente o planeta de modo euclidiano e não é difícil comprovar tal afirmação, basta observar que ao demonstrar o comportamento da gravidade sobre os seres humanos, evidenciam modelos euclidianos em detrimento a modelos esféricos, hiperbólicos e até elipsóidicos.

\section{O posicionamento dos Parâmetros Curriculares Nacionais ( $\mathrm{PCN})$ sobre o ensino de outros sistemas geométricos}

A Geometria é um dos temas mais interessantes para ser explorada pelos professores, por se constituir de uma riqueza em ilustrações, por possibilitar resoluções diversas com criatividade, e por fim, proporcionar aos estudantes uma interação mais dinâmica com o conhecimento. Uma das justificativas para esta afirmação é a existência de discussões acerca da inserção de Geometria Esférica e Hiperbólica nos currículos escolares que se encontram nos Parâmetros Curriculares Nacionais de Matemática (BRASIL, 1998), os quais apresentam a importância do ensino de outras geometrias aos estudantes:

\footnotetext{
[...] a Matemática não evolui de forma linear e logicamente organizada. Desenvolve-se com movimentos de idas e vindas, com rupturas de paradigmas. Frequentemente um conhecimento é amplamente utilizado na ciência ou na tecnologia antes de ser incorporado a um dos sistemas lógicos formais do corpo da Matemática. Exemplos desse fato podem ser encontrados no surgimento dos números negativos, irracionais e imaginários. Uma instância importante de mudança de paradigma ocorreu quando se superou a visão de uma única geometria do real, a Geometria Euclidiana, para aceitação de uma pluralidade de modelos geométricos, logicamente consistentes, que podem modelar a realidade do espaço físico. (BRASIL, 1998, p. 24, grifo nosso).
}

Por vezes, essa concepção linear faz com que, ao se definir qual será o elo inicial da cadeia, tomem-se os chamados fundamentos como ponto de partida. É o que ocorre, por exemplo, quando se privilegiam as noções de ponto, reta e plano, como referência inicial para o ensino de Geometria ou quando se tomam os conjuntos como base para a aprendizagem de números e operações, caminhos que não são necessariamente os mais adequados. O que também se observa em termos escolares é que muitas vezes os conteúdos geométricos são tratados isoladamente e são apresentados e exauridos num único momento. Quando acontece de serem retomados (geralmente num mesmo nível de aprofundamento, apoiando-se nos mesmos recursos), é apenas com a perspectiva de utilizá-los como ferramentas para a aprendizagem de novas noções e não para possibilitar a inserção de novas ideias. 


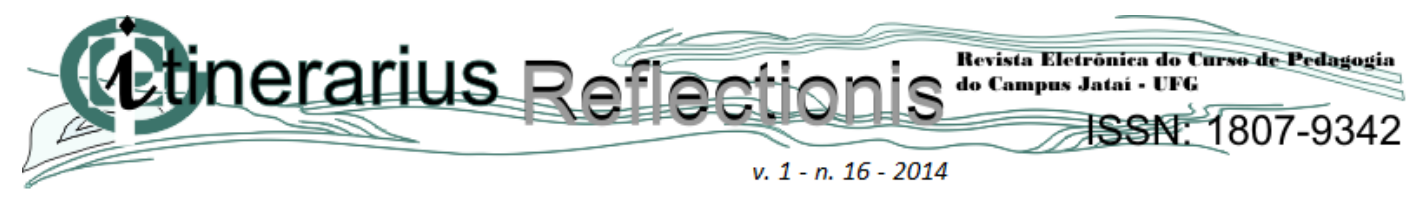

Neste sentido, os PCN (Parâmetros Curriculares Nacionais) colocam que entre os objetivos do ensino de Matemática, se encontra o desenvolvimento do pensamento geométrico. Recomenda-se a exploração de situações de aprendizagem que levem o estudante a resolver situações problema de localização e deslocamento de pontos no espaço, ler mapas, estimar e comparar distâncias percorridas, reconhecer propriedades de formas geométricas e saber usar diferentes unidades de medida. Portanto, há uma orientação para situações de aprendizagem que levem o estudante a estabelecer diferenças entre objetos sob diferentes pontos de vista, construindo e interpretando suas representações.

Para estabelecer diferenças entre objetos geométricos, conforme orienta os PCN, é preciso identificar uma pluralidade de modelos geométricos na natureza que pode ser pelo estudo das navegações, no sistema de localização por GPS (Sistema de Posicionamento Global), em aulas de Geografia ao tratar sobre o planeta Terra, na Física para compreensão do comportamento da luz no espaço, em objetos comuns do nosso cotidiano, como trompetes, batatas enlatadas, cornetas, ondas do mar, poltronas, garrafas de vinhos e outros que transitam nas diversas áreas do conhecimento. Com tantas possibilidades citadas e diversas pesquisas realizadas, porque grande parte dos currículos escolares se encontra ainda pautados em conhecimentos euclidianos? Essa pergunta promove discussões entre pesquisadores, como Kalleff (2004) e Cabariti (2006), no entanto, uma apresentação de situações cujas resoluções necessitem de conhecimentos geométricos esféricos e hiperbólicos a partir de um resgate histórico, configura materiais potenciais para o objetivo a qual este trabalho se dispõe: uma aprendizagem significativa sobre Geometria Esférica e Hiperbólica no Ensino Médio.

Para Coutinho (2001), a abordagem de outras geometrias em sala de aula, conforme orientações dos PCN, possibilita relacionar a Geometria a um campo atual e ativo de pesquisa científica que vai além das ideias apresentadas por Euclides. Nessa direção, é preciso uma compreensão por parte dos professores e pesquisadores que a Geometria Euclidiana não é a única possível, praticável no mundo e que solucionará todos os problemas no cotidiano e do mundo cientifico.

\section{Alguns problemas que impedem a inserção efetiva de conteúdos de Geometria Esférica e Hiperbólica no Ensino Médio}




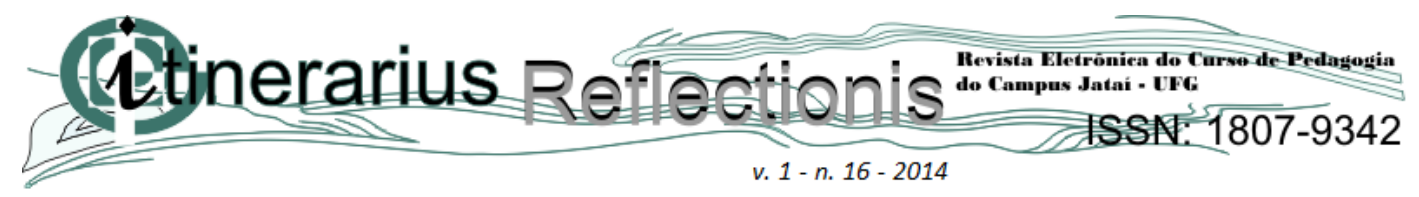

A Geometria é considerada como um dos alicerces da Matemática, porém, como é abordada na maioria das escolas atualmente, por meio de problemas descontextualizados, por métodos de repetição sem relacionar com o cotidiano do estudante, parece não manifestar na maioria dos estudantes grande interesse para sua aprendizagem. Muitos professores e a maioria dos estudantes acabam valorizando regras e exemplos de procedimentos para serem utilizados em problemas, que em geral, são superficiais. Para Coll, Marchesi e Palácios (2007) e Coll et al (2012), a aprendizagem repetitiva não busca relacionar os conhecimentos prévios com o novo conteúdo apresentado.

A ausência de uma abordagem sobre Geometria Esférica e Hiperbólica no Ensino Médio mostra que a maioria dos professores de Matemática desconsidera os conhecimentos cotidianos dos estudantes ou como afirma Demo (2012), seguem a metodologia de um determinado livro didático como fonte única de conhecimento, o que evidencia uma fragilidade de um sistema de ensino que privilegia resultados de modo mecânico e sem relação com o cotidiano.

Chassot (2011) afirma que a luta é para tornar o ensino menos asséptico, menos peremptório, menos abstrato, menos a-histórico e menos ferreteador. Por se tratar de um assunto pouco conhecido entre a maioria dos professores do Ensino Médio, as evidências mostram que, entre outros fatores que inviabilizam a introdução de Geometria Esférica e Hiperbólica no ensino escolar, está a complexidade matemática exigida por este tema, tornando esta área bastante técnica até mesmo no meio acadêmico. Porém, a história da Educação mostra, que muitos conteúdos como números complexos, polinômios e Geometria Analítica, já foram considerados de difícil abordagem na escola e atualmente constam sem qualquer questionamento nos currículos de Matemática.

Apesar de sua reconhecida importância porque a Geometria é deixada em segundo plano ou até muitas vezes é desprezada pelos professores? Uma possível resposta entre muitas razões, está na promulgação da primeira LDB (Lei de Diretrizes e Bases do Ensino) de $1^{\circ}$ e $2^{\circ}$ Graus em 1971, que possibilitou a cada professor elaborar seu programa de acordo com as necessidades dos estudantes. É possível que uma formação inicial deficitária de diversos professores acerca dos conhecimentos de Geometria Esférica e Hiperbólica também seja um indicativo para ausência deste tema 
em sala de aula. O fato é que muitos professores se tornaram dependentes dos livros didáticos, em que estes enfatizam conteúdos algébricos em detrimento dos conhecimentos geométricos. Para Vogelmann (2011) e Pozo (1998), a maioria dos professores e os livros didáticos formam um pano de fundo para a Aritmética e a Álgebra, deixando a Geometria em segundo plano.

Para Almouloud (2004), apesar da Geometria Esférica e Hiperbólica ser um ramo importante da Matemática, por servir principalmente de instrumento para diversas áreas do conhecimento, existe atualmente problemas relacionados ao seu ensino, caracterizadas pela falta de correlação com outras áreas do conhecimento, o que impede uma visão mais ampla e crítica por parte do estudante. Por outro lado, é preciso lembrar que muito se tem discutido e pesquisado sobre o ensino de Geometria na Educação Básica.

Existe, na verdade, um excesso de formalismo com a prevalência das demonstrações geométricas euclidianas e o abandono por completo da Geometria Esférica em sala de aula. Embora a Geometria de Euclides, por ser pouco privilegiada pelos professores em suas aulas, pelo rigor de suas demonstrações e por ser facilmente aceita pelos nossos sentidos, se caracterize como um possível modelo para a maioria das Ciências, em detrimento a Geometria Esférica e Hiperbólica.

\section{Algumas justificativas para apresentação de conceitos elementares de Geometria Esférica e Hiperbólica no Ensino Médio}

Atualmente, os conteúdos geométricos ensinados nas escolas possibilitam a resolução de problemas para superfícies planas, como por exemplo, quadrado, retângulo, triângulo, círculos e para sólidos de revolução como cone, cilindro, pirâmide e esfera. Contudo, existem objetos que se encontram na natureza que exigem conhecimentos que transcendem a Geometria Euclidiana.

Para Postman (2002), mesmo os conhecimentos que estão corretos e parece não precisar de emenda, estão limitados em seu alcance e sua aplicabilidade. Por exemplo, é possível citar o caso de um triângulo que em um plano possui invariavelmente uma soma de $180^{\circ}$ para seus ângulos internos, porém, em uma superfície curva, isto não é totalmente verdade. Dessa maneira, os conhecimentos geométricos organizados por Euclides são limitados, no sentido de explicar determinadas situações no campo da Geometria. 
Um dos pontos mais notáveis para a aplicação de conhecimentos geométricos não euclidianos é a prática da navegação, em que certamente a curvatura da Terra não pode ser desprezada. Ainda existem pessoas com a sensação de que um navio ao realizar determinada viagem entre dois países sua trajetória será em linha reta, contudo, devido ao fato do navio acompanhar a curvatura da Terra, o caminho descrito será um arco, demonstrando que a menor distância entre dois pontos pode não ser um segmento de reta.

Pelo fato da Geometria Euclidiana não ser a única aplicável a situações do cotidiano, talvez seja coerente, segundo Paty (2012) e Cedrez (2012), pensar que as diferentes geometrias ocupem lugares próprios no universo matemático, e se constituem, instrumentos de conhecimento ajustados a determinados problemas. Dessa maneira, é impossível elencar qual delas é a melhor ou mais útil, mas é possível definir qual delas deve ser utilizada. Por exemplo, os conhecimentos euclidianos são adequados ao trabalho do pedreiro que utiliza instrumentos como régua e esquadro para realizar marcações e delimitar áreas de construção, não ocorrendo com um marinheiro ou um piloto de avião que realiza viagens entre dois países.

Com relação a sua aplicação em sala de aula, o ensino de Geometria Esférica e Hiperbólica há quase três décadas se manifesta em algumas propostas curriculares brasileiras, como por exemplo, no Currículo Básico da Prefeitura Municipal de Curitiba (1988) ou na Proposta Curricular para a Matemática do Ensino Fundamental de São Paulo (1991, p. 88) que sugere o ensino da Geometria Esférica para “[...] concretizar as noções de círculos máximos e circunferências máximas, respectivamente, em esferas e superfícies esféricas [...]".

Contudo, discussões sobre o ensino de noções básicas de Geometria Esférica e Hiperbólica ainda não aconteceu nas escolas da rede pública de muitos estados brasileiros, possivelmente justificado pela dificuldade de sua compreensão por parte de alguns professores, pelo desconhecimento de linhas metodológicas ou, ainda, pela ausência de textos nos livros didáticos que contemplem o assunto.

Sob o olhar da aprendizagem de Geometria, os estudantes, em geral, têm acentuadas dificuldades em resolver problemas que transitam no campo dos conceitos geométricos. As fragilidades que os estudantes evidenciam na interpretação e solução de situações do cotidiano, nos quais são necessários conhecimentos geométricos é um dos 
grandes desafios enfrentados pelos professores. Uma abordagem de Geometria Esférica e Hiperbólica por meio de diferentes atividades é uma possível hipótese para o estudante realizar uma leitura do mundo mais concisa e universal e é nessa direção que este artigo justifica sua importância.

\section{Considerações}

A Matemática desenvolveu-se seguindo caminhos diferentes nas diversas culturas. O modelo de Geometria hoje mais utilizado nas escolas, originou-se com a civilização grega, no período que vai aproximadamente de 700 a.C. a 300 d.C., abrigando sistemas formais, logicamente estruturados a partir de um conjunto de premissas e empregando regras de raciocínio preestabelecidas. A maturidade desses sistemas formais foi atingida no século XIX, com o surgimento da Geometria Esférica e Hiperbólica.

Os conceitos elementares de Geometria constituem parte importante do currículo de Matemática no Ensino Médio, porque, por meio deles, o estudante desenvolve um tipo especial de pensamento que lhe permite compreender, descrever e representar, de forma organizada, o mundo em que vive. O estudo da Geometria Esférica e Hiperbólica é um campo fértil para trabalhar com situações-problema e é um tema pelo qual os estudantes costumam se interessar naturalmente. O trabalho com conceitos elementares no campo da Geométrica Esférica e Hiperbólica contribui para a aprendizagem também de números e medidas, pois estimula o estudante a observar, perceber semelhanças e diferenças, identificar regularidades etc.

A investigação do espaço esférico e hiperbólico por parte do professor, pressupõe a exploração de situações em que sejam necessárias algumas construções geométricas com régua e compasso, como visualização e aplicação de propriedades das figuras, além da construção de outras relações. Esta amálgama de conteúdos, ainda pouco exploradas na escola, contempla não apenas o estudo das formas, mas também as noções relativas a posição, localização de figuras e deslocamentos no plano e sistemas de coordenadas.

Deve destacar-se também a importância das transformações geométricas (isometrias, homotetias) nestes modelos geométricos, de modo que permita o desenvolvimento de habilidades de percepção espacial e como recurso para induzir de 


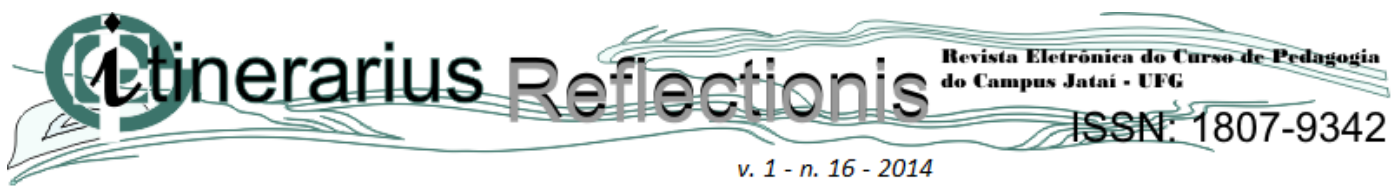

forma experimental a descoberta, por exemplo, das condições para que duas figuras sejam congruentes ou semelhantes. Além disso, o espaço deve ser explorado a partir de objetos do mundo físico, de obras de arte, pinturas, desenhos, esculturas e artesanato, de modo que permita ao estudante estabelecer conexões entre a Geometria Esférica e Hiperbólica com outras áreas do conhecimento.

\section{Referências}

ALMOULOUD, S. A. A geometria no ensino fundamental: reflexões sobre uma experiência de formação envolvendo professores e alunos. Revista Brasileira de Educação, São Paulo, n. 27, p. 94 - 108, Set /Out /Nov /Dez 2004.

ALVES, S. Geometria Não Euclidiana. São Paulo: IME-USP: material para oficina; Semana da Licenciatura, 2008.

BOYER, C. B. História da Matemática. 2a . ed. São Paulo: Blücher, 2009.

BONGIOVANI, V. De Euclides às geometrias não euclidianas. Revista Iberoamericana de Educación Matemática. São Paulo, v.1, n. 22, p. 37-51, 2010.

BRASIL. Secretaria de Educação Fundamental. Parâmetros curriculares nacionais: matemática. Brasília: MEC/SEF, 1998.

CABARITI, E. A geometria hiperbólica na formação docente: possibilidades de uma proposta com o auxílio do cabri-géomètre. III Seminário Internacional de Pesquisa em Educação Matemática, 2006, São Paulo.

CARVAlHO, M. A. S.; CARVAlHO, A. M. F. T. C. O ensino de geometria não euclidiana na educação básica. XIII Conferência Interamericana de Educação Matemática, 2011, Recife.

CEDREZ, A. J. P. Construcción, necessidad e intuición de essência em geometria. Scientia \& Studia. São Paulo, v. 7, n. 4, p. 595-617, 2012.

CHASSOT, A. A alfabetização científica: questões e desafios para a educação. Ijuí: Unijuí, 2011.

COLL, C., et al. O Construtivismo na Sala de Aula. São Paulo: Ática, 2012.

COLL, C.; MARCHESI, A.; PALACIOS, J. Desenvolvimento psicológico e educação: psicologia da educação escolar. $2^{\mathrm{a}}$. ed. Porto Alegre: Artmed, 2007.

COUTINHO, L. Convite às Geometrias Não Euclidianas. $2^{\mathrm{a}}$. ed. Rio de Janeiro: Interciência, 2001.

CURITIBA. Secretaria Municipal de Educação. Currículo básico: uma contribuição para a escola pública brasileira. Imprensa Oficial do Estado Paraná, 1988.

DEMO, P. O mais importante da educação importante. São Paulo: Atlas, 2012. 
EVES, H. Introdução à história da Matemática. São Paulo: Unicamp, 2008.

KALEFF, A. M. Desenvolvimento de Atividades Introdutórias ao Estudo das Geometrias não Euclidianas: Atividades Interdisciplinares para Sala de Aula e Museus Interativos. Congresso Brasileiro de Extensão Universitária, n. 2, 2004. Belo Horizonte.

LEIVAS, J. C. P. Educação geométrica: reflexões sobre ensino e aprendizagem em geometria. Revista SBEM-RS, Porto Alegre, n. 13, v.1, p. 9-16, 2012.

MARTOS, Z. G. Geometrias não euclidianas: uma proposta metodológica para o ensino de Geometria no Ensino Fundamental. 143f. Dissertação (Mestrado em Educação Matemática) - Instituto de Geociências e Ciências exatas, Universidade Estadual Paulista, Rio Claro, 2012.

MLODINOW, L. A janela de Euclides: a história da geometria, das linhas paralelas ao hiperespaço. São Paulo: Geração, 2010.

POSTMAN, N. O fim da educação: redefinindo o valor da escola. Rio de Janeiro: Graphia, 2002.

PATY, M. O estilo cientifico de Einstein na exploração do domínio quântico: uma visão da relação entre a teoria e seu objeto. Scientia \& Studia. v. 3, n. 4, p. 597-619, 2012.

POZO, J. I. Teorias cognitivas da aprendizagem. $3^{\mathrm{a}}$. ed. São Paulo: Artes Medicas, 1998.

SÃO PAULO. Secretaria de Educação. Coordenadoria de Estudos e Normas Pedagógicas. Proposta curricular para o ensino de Matemática do $1 .^{\circ}$ grau. $4^{\mathrm{a}}$. ed. São Paulo: SE/CENP, 1991.

VOGELMANN, E. P. A arte de ensinar e construir o conhecimento. São Paulo: Saraiva, 2011. 